\title{
Recurrence formulas for Macdonald polynomials of type $A$
}

\author{
Michel Lassalle • Michael J. Schlosser
}

Received: 12 February 2009 / Accepted: 9 October 2009 / Published online: 13 November 2009

(C) Springer Science+Business Media, LLC 2009

\begin{abstract}
We consider products of two Macdonald polynomials of type $A$, indexed by dominant weights which are respectively a multiple of the first fundamental weight and a weight having zero component on the $k$ th fundamental weight. We give the explicit decomposition of any Macdonald polynomial of type $A$ in terms of this basis.
\end{abstract}

Keywords Macdonald polynomials $\cdot$ Pieri formula $\cdot$ Multidimensional matrix inverse

\section{Introduction}

In the 1980s, Macdonald [6-8] introduced a class of orthogonal polynomials which are Laurent polynomials in several variables and generalize the Weyl characters of compact simple Lie groups. In the simplest situation, given a root system $R$, these polynomials are elements of the group algebra of the weight lattice of $R$, indexed by the dominant weights and depending on two parameters $(q, t)$.

When $R$ is of type $A_{n}$, these Macdonald polynomials are in bijective correspondence with the symmetric functions $\mathcal{P}_{\lambda}(q, t)$ indexed by partitions, introduced by Macdonald some years before [4, 5]. In fact, they correspond to $\mathcal{P}_{\lambda}(q, t)\left(x_{1}, \ldots, x_{n+1}\right)$, for a partition $\lambda=\left(\lambda_{1}, \ldots, \lambda_{n}\right)$ of length $n$, with the $n+1$ variables $\left(x_{1}, \ldots, x_{n+1}\right)$ linked by the condition $x_{1} \cdots x_{n+1}=1$.

M. Lassalle $(\bowtie)$

Centre National de la Recherche Scientifique, Institut Gaspard-Monge,

Université de Marne-la-Vallée, 77454 Marne-la-Vallée Cedex, France

e-mail: lassalle@univ-mlv.fr

url: http://igm.univ-mlv.fr/ lassalle

M.J. Schlosser

Fakultät für Mathematik, Universität Wien, Nordbergstraße 15, 1090 Vienna, Austria

e-mail: michael.schlosser@univie.ac.at

url: http://www.mat.univie.ac.at/ schlosse 
The purpose of this article is to extend the result of [3], given for the symmetric functions $\mathcal{P}_{\lambda}(q, t)$, to the framework of the root system $A_{n}$.

More precisely, in [3, Theorem 4.1] we obtained a recurrence formula giving the symmetric function $\mathcal{P}_{\left(\lambda_{1}, \ldots, \lambda_{n}\right)}(q, t)$ as a sum

$$
\mathcal{P}_{\left(\lambda_{1}, \ldots, \lambda_{n}\right)}=\sum_{\theta \in \mathbb{N}^{n-1}} C_{\theta_{1}, \ldots, \theta_{n-1}} \mathcal{P}_{\left(\lambda_{1}+\theta_{1}, \ldots, \lambda_{n-1}+\theta_{n-1}\right)} \mathcal{P}_{\lambda_{n}-|\theta|}
$$

with $|\theta|=\sum_{i=1}^{n-1} \theta_{i}$ and $\mathbb{N}$ the set of nonnegative integers. This formula was obtained by inverting the "Pieri formula," which conversely expresses the product $\mathcal{P}_{\left(\lambda_{1}, \ldots, \lambda_{n-1}\right)} \mathcal{P}_{\lambda_{n}}$ as a sum

$$
\mathcal{P}_{\left(\lambda_{1}, \ldots, \lambda_{n-1}\right)} \mathcal{P}_{\lambda_{n}}=\sum_{\theta \in \mathbb{N}^{n-1}} c_{\theta_{1}, \ldots, \theta_{n-1}} \mathcal{P}_{\left(\lambda_{1}+\theta_{1}, \ldots, \lambda_{n-1}+\theta_{n-1}, \lambda_{n}-|\theta|\right)}
$$

Both expansions are identities between symmetric functions, valid for any number of variables.

These identities may also be written in terms of Macdonald polynomials of type $A_{n}$. For this purpose, let $\left\{\omega_{i}, 1 \leq i \leq n\right\}$ be the $n$ fundamental weights of the root system $A_{n}$. Let $P_{\lambda}$ denote the Macdonald polynomial associated with the dominant weight $\lambda=\sum_{i=1}^{n} \lambda_{i} \omega_{i}$. The recurrence formula (1.1), written for $n+1$ variables $\left(x_{1}, \ldots, x_{n+1}\right)$ linked by $x_{1} \cdots x_{n+1}=1$, yields

$$
P_{\lambda}=\sum_{\theta \in \mathbb{N}^{n-1}} C_{\theta_{1}, \ldots, \theta_{n-1}} P_{\left(\lambda_{n}-|\theta|\right) \omega_{1}} P_{\mu}
$$

with $\mu=\sum_{i=1}^{n-2}\left(\lambda_{i}+\theta_{i}-\theta_{i+1}\right) \omega_{i}+\left(\lambda_{n-1}+\lambda_{n}+\theta_{n-1}\right) \omega_{n-1}$. This alternative formulation is obvious and does not bring anything new.

However the method of [3], when applied in the $A_{n}$ root system framework, allows us to get a much stronger result. Indeed, let $k$ be a fixed integer with $1 \leq k \leq n$. In this paper we shall write the Macdonald polynomial $P_{\lambda}$ in terms of products $P_{r \omega_{1}} P_{\mu}$ with $\mu=\sum_{i=1}^{n} \mu_{i} \omega_{i}$ and $\mu_{k}=0$. There are $n$ such recurrence formulas, (1.2) being the particular case $k=n$ of the latter.

This paper is organized as follows. In Sect. 2 we introduce our notation for the root system $A_{n}$ and recall general facts about the corresponding Macdonald polynomials. Their Pieri formula, which involves a specific infinite-multidimensional matrix, is studied in Sect. 3, starting from the one given by Macdonald for the symmetric functions $\mathcal{P}_{\lambda}(q, t)$ [5, p. 340]. In Sect. 4 we invert the Pieri matrix by applying a particular multidimensional matrix inverse, given separately in the Appendix. This matrix inverse is equivalent to one previously obtained in [3, Sect. 2] by using operator methods. As a result of inverting the Pieri formula, we obtain recurrence formulas for $A_{n}$ Macdonald polynomials. Finally, in Sect. 5 we detail the examples of the $A_{2}$ and $A_{3}$ cases and compare them to earlier results. 


\section{Macdonald polynomials of type $A$}

The standard references for Macdonald polynomials associated with root systems are $[6-8]$.

Let us consider the space $\mathbb{R}^{n+1}$ endowed with the usual scalar product and the quotient space $V=\mathbb{R}^{n+1} / \mathbb{R}(1, \ldots, 1)$, where $\mathbb{R}(1, \ldots, 1)$ is the subspace spanned by the vector $(1, \ldots, 1)$. Let $\varepsilon_{1}, \ldots, \varepsilon_{n+1}$ denote the images in $V$ of the coordinate vectors of $\mathbb{R}^{n+1}$, linked by $\sum_{i=1}^{n+1} \varepsilon_{i}=0$.

The root system of type $A_{n}$ is formed by the vectors $\left\{\varepsilon_{i}-\varepsilon_{j}, i \neq j\right\}$. The positive roots are $\left\{\varepsilon_{i}-\varepsilon_{j}, i<j\right\}$, and the simple roots are $\varepsilon_{i}-\varepsilon_{i+1}$ for $1 \leq i \leq n$. The Weyl group is the symmetric group $W=S_{n+1}$ acting by permutation of the coordinates.

The weight lattice $P$ is formed by integral linear combinations of the fundamental weights $\left\{\omega_{i}, 1 \leq i \leq n\right\}$ defined by $\omega_{i}=\varepsilon_{1}+\cdots+\varepsilon_{i}$. Let $\omega_{i}=0$ for $i=0, n+1$. We denote by $P^{+}$the set of dominant weights $\lambda=\sum_{i=1}^{n} \lambda_{i} \omega_{i}$, which are nonnegative integral linear combinations of the fundamental weights.

There is the following correspondence between dominant weights and partitions. Given a dominant weight, if we write it as

$$
\lambda=\sum_{i=1}^{n} \lambda_{i} \omega_{i}=\sum_{i=1}^{n+1} \mu_{i} \varepsilon_{i}
$$

the sequence $\mu=\left(\mu_{1}, \ldots, \mu_{n+1}\right)$ is a partition with length $\leq n+1$. We have

$$
\lambda_{i}=\mu_{i}-\mu_{i+1} \quad \text { and } \quad \mu_{i}=\mu_{n+1}+\sum_{j=i}^{n} \lambda_{j} .
$$

Thus $\mu$ is defined up to $\mu_{n+1}$, and two partitions $\mu, v$ correspond to the same weight $\lambda$ if and only if $\mu_{1}-v_{1}=\cdots=\mu_{n+1}-v_{n+1}$. We denote by $\mathcal{C}_{\lambda}$ the family of partitions thus defined.

Let $A$ denote the group algebra over $\mathbb{R}$ of the free Abelian group $P$. For each $\lambda \in P$, let $e^{\lambda}$ denote the corresponding element of $A$, subject to the multiplication rule $e^{\lambda} e^{\mu}=e^{\lambda+\mu}$. The set $\left\{e^{\lambda}, \lambda \in P\right\}$ forms an $\mathbb{R}$-basis of $A$.

The Weyl group $W=S_{n+1}$ acts on $P$ and on $A$. Let $W \lambda$ denote the orbit of $\lambda \in P$ and $A^{W}$ the subspace of $W$-invariants in $A$. There are two important bases of $A^{W}$, both indexed by dominant weights. The first one is given by the orbit-sums

$$
m_{\lambda}=\sum_{\mu \in W \lambda} e^{\mu}
$$

The second one is provided by the Weyl characters

$$
\chi_{\lambda}=\delta^{-1} \sum_{w \in W} \operatorname{det}(w) e^{w(\lambda+\rho)}
$$

with $\rho=\sum_{i=1}^{n}(n-i+1) \varepsilon_{i}$ and $\delta=\sum_{w \in W} \operatorname{det}(w) e^{w(\rho)}$. The Macdonald polynomials $\left\{P_{\lambda}, \lambda \in P^{+}\right\}$form another basis defined as the eigenvectors of a specific selfadjoint operator (which we do not describe here). 
For $1 \leq i \leq n+1$, define $x_{i}=e^{\varepsilon_{i}}$, so that the variables $x_{i}$ are linked by $x_{1} \cdots x_{n+1}=1$. Then $\delta$ is the Vandermonde determinant $\prod_{i<j}\left(x_{i}-x_{j}\right)$. There is a correspondence between $A^{W}$ and the symmetric polynomials restricted to $n+1$ variables $x=\left(x_{1}, \ldots, x_{n+1}\right)$ linked by the previous condition.

In terms of bases this correspondence may be described as follows. Let $\lambda$ be any dominant weight, and let $x_{1} \cdots x_{n+1}=1$. All monomial symmetric functions $m_{\mu}\left(x_{1}, \ldots, x_{n+1}\right)$ with $\mu \in \mathcal{C}_{\lambda}$ are equal, and their common value is the orbitsum $m_{\lambda}$. Similarly, the Weyl character $\chi_{\lambda}$ is the common value of the Schur functions $s_{\mu}\left(x_{1}, \ldots, x_{n+1}\right), \mu \in \mathcal{C}_{\lambda}$, whereas the Macdonald polynomial $P_{\lambda}$ is the common value of the symmetric polynomials $\mathcal{P}_{\mu}(q, t)\left(x_{1}, \ldots, x_{n+1}\right)$ with $\mu \in \mathcal{C}_{\lambda}$ and $\mathcal{P}_{\mu}(q, t)$ the symmetric function studied in Chap. 6 of [5].

Given a positive integer $r$ and a dominant weight $\lambda$, the "Pieri formula" expands the product

$$
P_{r \omega_{1}} P_{\lambda}=\sum_{\rho} c_{\rho} P_{\lambda+\rho}
$$

in terms of Macdonald polynomials, where the range of $\rho$ and the values of the coefficients $c_{\rho}$ are to be determined.

Let $Q$ denote the root lattice spanned by the simple roots. For any vector $\tau$, define

$$
\Sigma(\tau)=C(\tau) \cap(\tau+Q)
$$

with $C(\tau)$ the convex hull of the Weyl group orbit of $\tau$. Since the orbit of $\omega_{1}=\varepsilon_{1}$ is the set $\left\{\varepsilon_{i}=\omega_{i}-\omega_{i-1}, 1 \leq i \leq n+1\right\}$, it is clear that $\Sigma\left(r \omega_{1}\right)$ is formed by vectors

$$
\sum_{i=1}^{n+1} \theta_{i}\left(\omega_{i}-\omega_{i-1}\right)=\sum_{i=1}^{n}\left(\theta_{i}-\theta_{i+1}\right) \omega_{i}
$$

with $\theta=\left(\theta_{1}, \ldots, \theta_{n+1}\right) \in \mathbb{N}^{n+1}$ and $|\theta|=\sum_{i=1}^{n+1} \theta_{i}=r$.

By general results $[8,(5.3 .8)$, p. 104], it is known that the sum on the righthand side of the Pieri formula is restricted to vectors $\rho$ such that $\rho \in \Sigma\left(r \omega_{1}\right)$ and $\lambda+\rho \in P^{+}$. In the next section we shall give a direct proof of this result and make the value of the coefficient $c_{\rho}$ explicit.

\section{Pieri formula}

Let $0<q<1$. For any integer $r$, the classical $q$-shifted factorial $(u ; q)_{r}$ is defined by

$$
(u ; q)_{\infty}=\prod_{j \geq 0}\left(1-u q^{j}\right), \quad(u ; q)_{r}=(u ; q)_{\infty} /\left(u q^{r} ; q\right)_{\infty} .
$$

Let $u=\left(u_{1}, \ldots, u_{m}\right)$ be $m$ indeterminates, and $\theta=\left(\theta_{1}, \ldots, \theta_{m}\right) \in \mathbb{N}^{m}$. For clarity of display, throughout this paper, any time such a pair $(u, \theta)$ is given, we shall implicitly assume $m$ auxiliary variables $v=\left(v_{1}, \ldots, v_{m}\right)$ to be defined by $v_{i}=q^{\theta_{i}} u_{i}$.

Macdonald polynomials of type $A_{n}$ satisfy the following Pieri formula. 
Theorem 3.1 Let $\lambda=\sum_{i=1}^{n} \lambda_{i} \omega_{i}$ be a dominant weight, and $r \in \mathbb{N}$. For any $1 \leq i \leq$ $n+1$, define

$$
u_{i}=q^{\sum_{j=i}^{n} \lambda_{j}} t^{-i}
$$

and for $\theta \in \mathbb{N}^{n+1}$,

$$
d_{\theta}\left(u_{1}, \ldots, u_{n+1} ; r\right)=\frac{(q ; q)_{r}}{(t ; q)_{r}} \prod_{j=1}^{n+1} \frac{(t ; q)_{\theta_{j}}}{(q ; q)_{\theta_{j}}} \prod_{1 \leq i<j \leq n+1} \frac{\left(t v_{i} / v_{j} ; q\right)_{\theta_{j}}}{\left(q v_{i} / v_{j} ; q\right)_{\theta_{j}}} \frac{\left(q u_{i} / t v_{j} ; q\right)_{\theta_{j}}}{\left(u_{i} / v_{j} ; q\right)_{\theta_{j}}}
$$

We have

$$
P_{r \omega_{1}} P_{\lambda}=\sum_{\substack{\theta \in \mathbb{N}^{n+1} \\|\theta|=r}} d_{\theta}\left(u_{1}, \ldots, u_{n+1} ; r\right) P_{\lambda+\rho}
$$

with $\rho=\sum_{i=1}^{n}\left(\theta_{i}-\theta_{i+1}\right) \omega_{i}$.

Proof In a first step, we write the Pieri formula for arbitrary $\mathcal{P}_{\mu}(q, t)$ with $\mu=$ $\left(\mu_{1}, \ldots, \mu_{n}\right)$ being a partition having length $\leq n$. We start from [5, p. 340, (6.24)(i)] and $\left[5\right.$, p. 342, Example 2(a)]. Replacing $g_{r}$ by $(t ; q)_{r} /(q ; q)_{r} \mathcal{P}_{(r)}$, we have

$$
\mathcal{P}_{(r)} \mathcal{P}_{\mu}=\sum_{\kappa \supset \mu} \varphi_{\kappa / \mu} \mathcal{P}_{\kappa}
$$

where the skew-diagram $\kappa-\mu$ is a horizontal $r$-strip, i.e., has at most one node in each column. The Pieri coefficient $\varphi_{\kappa / \mu}$ is given by

$$
\begin{aligned}
\frac{(t ; q)_{r}}{(q ; q)_{r}} \varphi_{\kappa / \mu} & =\prod_{1 \leq i \leq j \leq l(\kappa)} \frac{f\left(q^{\kappa_{i}-\kappa_{j}} t^{j-i}\right)}{f\left(q^{\kappa_{i}-\mu_{j}} t^{j-i}\right)} \frac{f\left(q^{\mu_{i}-\mu_{j+1}} t^{j-i}\right)}{f\left(q^{\mu_{i}-\kappa_{j+1}} t^{j-i}\right)} \\
& =\prod_{1 \leq i \leq j \leq l(\kappa)} \frac{w_{\kappa_{j}-\mu_{j}}\left(q^{\kappa_{i}-\kappa_{j}} t^{j-i}\right)}{w_{\kappa_{j+1}-\mu_{j+1}}\left(q^{\mu_{i}-\kappa_{j+1}} t^{j-i}\right)}
\end{aligned}
$$

with $f(u)=(t u ; q)_{\infty} /(q u ; q)_{\infty}$ and $w_{s}(u)=(t u ; q)_{s} /(q u ; q)_{s}$.

Since $\kappa-\mu$ is a horizontal strip, the length $l(\kappa)$ of $\kappa$ is at most equal to $n+1$, so we can write $\kappa=\left(\mu_{1}+\theta_{1}, \ldots, \mu_{n}+\theta_{n}, \theta_{n+1}\right)$ with $|\theta|=r$. Then

$$
\begin{aligned}
\frac{(t ; q)_{r}}{(q ; q)_{r}} \varphi_{\kappa / \mu} & =\prod_{1 \leq i \leq j \leq l(\kappa)} w_{\theta_{j}}\left(q^{\kappa_{i}-\kappa_{j}} t^{j-i}\right) \prod_{1 \leq i<j \leq l(\kappa)+1}\left(w_{\theta_{j}}\left(q^{\mu_{i}-\kappa_{j}} t^{j-i-1}\right)\right)^{-1} \\
& =\prod_{j=1}^{n+1} \frac{(t ; q)_{\theta_{j}}}{(q ; q)_{\theta_{j}}} \prod_{1 \leq i<j \leq n+1} \frac{\left(t v_{i} / v_{j} ; q\right)_{\theta_{j}}}{\left(q v_{i} / v_{j} ; q\right)_{\theta_{j}}} \frac{\left(q u_{i} / t v_{j} ; q\right)_{\theta_{j}}}{\left(u_{i} / v_{j} ; q\right)_{\theta_{j}}}
\end{aligned}
$$

where for $1 \leq i \leq n+1$, we set $u_{i}=q^{\mu_{i}} t^{-i}$ and $v_{i}=q^{\kappa_{i}} t^{-i}=q^{\theta_{i}} u_{i}$.

In a second step we translate this result in terms of $A_{n}$ Macdonald polynomials. Given the dominant weight $\lambda$, we choose $\mu=\left(\mu_{1}, \ldots, \mu_{n+1}\right)$ to be the unique 
element of $\mathcal{C}_{\lambda}$ such that $\mu_{n+1}=0$, i.e., with length $\leq n$. For $1 \leq i \leq n$, we have $\mu_{i}=\sum_{j=i}^{n} \lambda_{j}$. As for the partition $\kappa$ (with length $\leq n+1$ ), it belongs to $\mathcal{C}_{\sigma}$ with $\sigma=\sum_{k=1}^{n}\left(\kappa_{k}-\kappa_{k+1}\right) \omega_{k}=\sum_{k=1}^{n}\left(\lambda_{k}+\theta_{k}-\theta_{k+1}\right) \omega_{k}$. Hence the statement.

Remark On the right-hand side of the Pieri formula, the condition $\lambda+\rho \in P^{+}$is necessarily satisfied as soon as $d_{\theta}\left(u_{1}, \ldots, u_{n+1} ; r\right) \neq 0$. Using the correspondence between dominant weights and partitions, this may be verified on the Pieri formula

$$
\mathcal{P}_{(r)} \mathcal{P}_{\mu}=\sum_{\kappa=\left(\mu_{1}+\theta_{1}, \ldots, \mu_{n}+\theta_{n}, \theta_{n+1}\right)} \varphi_{\kappa / \mu} \mathcal{P}_{\kappa}
$$

We only have to show that $\varphi_{\kappa / \mu}$ necessarily vanishes when the multiinteger $\kappa$ is not a partition. But then there is an index $i$ such that $\kappa_{i}<\kappa_{i+1}$, so that the factor $\left(q u_{i} / t v_{i+1} ; q\right)_{\theta_{i+1}}$ in $\varphi_{\kappa / \mu}$ writes out as

$$
\left(1-q^{1+\mu_{i}-\kappa_{i+1}}\right) \cdots\left(1-q^{\mu_{i}-\mu_{i+1}}\right) .
$$

Due to $\kappa_{i}<\kappa_{i+1}$, this product would be $\neq 0$ only if $\mu_{i}<\mu_{i+1}$, which is impossible since $\mu$ is a partition.

From now on, we fix some integer $1 \leq k \leq n$. Substituting $r-|\theta|$ for $\theta_{k}$, the Pieri formula may be written in the more explicit form

$$
P_{r \omega_{1}} P_{\lambda}=\sum_{\theta=\left(\theta_{1}, \ldots, \theta_{k-1}, 0, \theta_{k+1}, \ldots, \theta_{n+1}\right) \in \mathbb{N}^{n}} \hat{d}_{\theta \mid \leq r} \hat{d}_{\theta}\left(u_{1}, \ldots, u_{n+1} ; r\right) P_{\lambda+\rho}
$$

with

$$
\rho=\sum_{\substack{1 \leq i \leq n \\ i \neq k-1, k}}\left(\theta_{i}-\theta_{i+1}\right) \omega_{i}+\theta_{k-1} \omega_{k-1}+(r-|\theta|)\left(\omega_{k}-\omega_{k-1}\right)-\theta_{k+1} \omega_{k}
$$

and

$$
\begin{aligned}
\hat{d}_{\theta}\left(u_{1}, \ldots, u_{n+1} ; r\right)= & \frac{(q ; q)_{r}}{(t ; q)_{r}} \frac{(t ; q)_{r-|\theta|}}{(q ; q)_{r-|\theta|}} \prod_{\substack{j=1 \\
j \neq k}}^{n+1} \frac{(t ; q)_{\theta_{j}}}{(q ; q)_{\theta_{j}}} \\
& \times \prod_{\substack{1 \leq i<j \leq n+1 \\
j \neq k}} \frac{\left(t v_{i} / v_{j} ; q\right)_{\theta_{j}}}{\left(q v_{i} / v_{j} ; q\right)_{\theta_{j}}} \frac{\left(q u_{i} / t v_{j} ; q\right)_{\theta_{j}}}{\left(u_{i} / v_{j} ; q\right)_{\theta_{j}}} \\
& \times \prod_{i=1}^{k-1} \frac{\left(t v_{i} / v_{k} ; q\right)_{r-|\theta|}}{\left(q v_{i} / v_{k} ; q\right)_{r-|\theta|}} \frac{\left(q u_{i} / t v_{k} ; q\right)_{r-|\theta|}}{\left(u_{i} / v_{k} ; q\right)_{r-|\theta|}}
\end{aligned}
$$

Here $u_{i}, v_{i}(1 \leq i \leq n+1)$ are as in Theorem 3.1, except $v_{k}=q^{r-|\theta|} u_{k}$. The sum is restricted to $|\theta| \leq r$ since $1 /(q ; q)_{s}=0$ for $s<0$. 
In a second step, we concentrate on the situation $\lambda_{k}=0$. Then each term on the right-hand side vanishes unless $\theta_{k+1}=0$. Indeed, if $\lambda_{k}=0$, one has $u_{k}=t u_{k+1}$ and $v_{k+1}=q^{\theta_{k+1}} u_{k+1}$. Hence, for $i=k$ and $j=k+1$, the factor $\left(q u_{i} / t v_{j} ; q\right)_{\theta_{j}}$ evaluates as

$$
\left(q u_{k} / t v_{k+1} ; q\right)_{\theta_{k+1}}=\left(q^{1-\theta_{k+1}} ; q\right)_{\theta_{k+1}}=\delta_{\theta_{k+1}, 0} .
$$

Therefore, if $\lambda_{k}=0$, the Pieri formula can be written as

$$
\begin{aligned}
& P_{r \omega_{1}} P_{\lambda} \\
& =\sum_{\theta=\left(\theta_{1}, \ldots, \theta_{k-1}, 0,0, \theta_{k+2}, \ldots, \theta_{n+1}\right) \in \mathbb{N}^{n-1} \mid \operatorname{lor}} \tilde{d}_{\theta}\left(u_{1}, \ldots, u_{k-1}, u_{k}, u_{k+2}, \ldots, u_{n+1} ; k, r\right) P_{\lambda+\rho}
\end{aligned}
$$

with

$$
\rho=\sum_{\substack{1 \leq i \leq n \\ i \neq k-1, k, k+1}}\left(\theta_{i}-\theta_{i+1}\right) \omega_{i}+\theta_{k-1} \omega_{k-1}+(r-|\theta|)\left(\omega_{k}-\omega_{k-1}\right)-\theta_{k+2} \omega_{k+1}
$$

and

$$
\begin{aligned}
\tilde{d}_{\theta}( & \left.u_{1}, \ldots, u_{k-1}, u_{k}, u_{k+2}, \ldots, u_{n+1} ; k, r\right) \\
= & \frac{(q ; q)_{r}}{(t ; q)_{r}} \frac{(t ; q)_{r-|\theta|}}{(q ; q)_{r-|\theta|}} \prod_{\substack{i=1 \\
i \neq k, k+1}}^{n+1} \frac{(t ; q)_{\theta_{i}}}{(q ; q)_{\theta_{i}}} \prod_{\substack{1 \leq i<j \leq n+1 \\
i \neq k, k+1 \\
j \neq k, k+1}} \frac{\left(t v_{i} / v_{j} ; q\right)_{\theta_{j}}}{\left(q v_{i} / v_{j} ; q\right)_{\theta_{j}}} \frac{\left(q u_{i} / t v_{j} ; q\right)_{\theta_{j}}}{\left(u_{i} / v_{j} ; q\right)_{\theta_{j}}} \\
& \times \prod_{i=1}^{k-1} \frac{\left(t v_{i} / v_{k} ; q\right)_{r-|\theta|}}{\left(q v_{i} / v_{k} ; q\right)_{r-|\theta|}} \frac{\left(q u_{i} / t v_{k} ; q\right)_{r-|\theta|}}{\left(u_{i} / v_{k} ; q\right)_{r-|\theta|}} \prod_{j=k+2}^{n+1} \frac{\left(t v_{k} / v_{j} ; q\right)_{\theta_{j}}}{\left(q v_{k} / v_{j} ; q\right)_{\theta_{j}}} \frac{\left(q u_{k} / t^{2} v_{j} ; q\right)_{\theta_{j}}}{\left(u_{k} / t v_{j} ; q\right)_{\theta_{j}}} .
\end{aligned}
$$

Here the notation is the same as before, including $v_{k}=q^{r-|\theta|} u_{k}$. For $j \geq k+2$, we have used

$$
\begin{aligned}
& \frac{\left(t v_{k} / v_{j} ; q\right)_{\theta_{j}}}{\left(q v_{k} / v_{j} ; q\right)_{\theta_{j}}} \frac{\left(q u_{k} / t v_{j} ; q\right)_{\theta_{j}}}{\left(t v_{k+1} / v_{j} ; q\right)_{\theta_{j}}} \frac{\left(q v_{\theta_{j}}\right.}{\left(q v_{k+1} / v_{j} ; q\right)_{\theta_{j}}} \frac{\left(q u_{k+1} / t v_{j} ; q\right)_{\theta_{j}}}{\left(u_{k+1} / v_{j} ; q\right)_{\theta_{j}}} \\
& =\frac{\left(t v_{k} / v_{j} ; q\right)_{\theta_{j}}}{\left(q v_{k} / v_{j} ; q\right)_{\theta_{j}}} \frac{\left(q u_{k} / t^{2} v_{j} ; q\right)_{\theta_{j}}}{\left(u_{k} / t v_{j} ; q\right)_{\theta_{j}}}
\end{aligned}
$$

which is a direct consequence of $v_{k+1}=u_{k+1}=u_{k} / t$.

In a third step, we perform some relabeling in order to remove the two 0's appearing in $\theta$. For that purpose, for $n$ indeterminates $\left(u_{0}, u_{1}, \ldots, u_{n-1}\right)$ and $\theta=$ $\left(\theta_{1}, \ldots, \theta_{n-1}\right) \in \mathbb{N}^{n-1}$, we define

$$
\begin{aligned}
& D_{\theta}\left(u_{0}, u_{1}, \ldots, u_{n-1} ; k, r\right) \\
& \quad=(q / t)^{|\theta|} \frac{\left(t^{2} u_{0} ; q\right)_{|\theta|}}{\left(q t u_{0} ; q\right)_{|\theta|}} \prod_{i=1}^{n-1} \frac{(t ; q)_{\theta_{i}}}{(q ; q)_{\theta_{i}}} \frac{\left(q^{|\theta|+1} u_{i} ; q\right)_{\theta_{i}}}{\left(q^{|\theta|} t u_{i} ; q\right)_{\theta_{i}}}
\end{aligned}
$$




$$
\begin{aligned}
& \times \prod_{1 \leq i<j \leq n-1} \frac{\left(t v_{i} / v_{j} ; q\right)_{\theta_{j}}}{\left(q v_{i} / v_{j} ; q\right)_{\theta_{j}}} \frac{\left(q u_{i} / t v_{j} ; q\right)_{\theta_{j}}}{\left(u_{i} / v_{j} ; q\right)_{\theta_{j}}} \\
& \times \prod_{i=1}^{k-1} \frac{\left(u_{i} / u_{0} ; q\right)_{\theta_{i}}}{\left(q u_{i} / t u_{0} ; q\right)_{\theta_{i}}} \frac{\left(q u_{i} / t u_{0} ; q\right)_{\theta_{i}-r+|\theta|}}{\left(u_{i} / u_{0} ; q\right)_{\theta_{i}}-r+|\theta|} \frac{\left(u_{i} / t u_{0} ; q\right)_{\theta_{i}-r+|\theta|}}{\left(q u_{i} / t^{2} u_{0} ; q\right)_{\theta_{i}-r+|\theta|}} \\
& \times \prod_{i=k}^{n-1} \frac{\left(t u_{i} / u_{0} ; q\right)_{\theta_{i}}}{\left(q u_{i} / u_{0} ; q\right)_{\theta_{i}}} .
\end{aligned}
$$

Lemma If we write

$$
w_{i}= \begin{cases}q^{-r} t^{-2}, & i=0 \\ q^{-r} u_{i} / t u_{k}, & 1 \leq i \leq k-1 \\ q^{-r} u_{i+2} / t u_{k}, & k \leq i \leq n-1\end{cases}
$$

we have

$$
\begin{aligned}
& D_{\theta}\left(w_{0}, w_{1}, \ldots, w_{n-1} ; k, r\right) \\
& \quad=\tilde{d}_{\left(\theta_{1}, \ldots, \theta_{k-1}, 0,0, \theta_{k}, \ldots, \theta_{n-1}\right)}\left(u_{1}, \ldots, u_{k-1}, u_{k}, u_{k+2}, \ldots, u_{n+1} ; k, r\right) .
\end{aligned}
$$

Proof Merely by substitution, and using $v_{k}=q^{r-|\theta|} u_{k}$, we only have to prove

$$
\begin{aligned}
(q / t)^{|\theta|} & \frac{\left(q^{-r} ; q\right)_{|\theta|}}{\left(q^{1-r} / t ; q\right)_{|\theta|}} \prod_{j=k+2}^{n+1} \frac{\left(q^{|\theta|-r+1} u_{j} / t u_{k} ; q\right)_{\theta_{j}}}{\left(q^{|\theta|-r} u_{j} / u_{k} ; q\right)_{\theta_{j}}} \frac{\left(t^{2} u_{j} / u_{k} ; q\right)_{\theta_{j}}}{\left(q t u_{j} / u_{k} ; q\right)_{\theta_{j}}} \\
\times & \prod_{i=1}^{k-1} \frac{\left(q^{|\theta|-r+1} u_{i} / t u_{k} ; q\right)_{\theta_{i}}}{\left(t u_{i} / u_{k} ; q\right)_{\theta_{i}}} \frac{\left(q^{|\theta|-r} u_{i} / u_{k} ; q\right)_{\theta_{i}}}{\left(q u_{i} / u_{k} ; q\right)_{\theta_{i}}} \\
& \times \prod_{i=1}^{k-1} \frac{\left(q u_{i} / u_{k} ; q\right)_{\theta_{i}-r+|\theta|}}{\left(t u_{i} / u_{k} ; q\right)_{\theta_{i}-r+|\theta|}} \frac{\left(u_{i} / u_{k} ; q\right)_{\theta_{i}-r+|\theta|}}{\left(q u_{i} / t u_{k} ; q\right)_{\theta_{i}-r+|\theta|}} \\
= & \frac{(q ; q)_{r}}{(t ; q)_{r}} \frac{(t ; q)_{r-|\theta|}}{(q ; q)_{r-|\theta|}} \prod_{i=1}^{k-1} \frac{\left(t v_{i} / q^{r-|\theta|} u_{k} ; q\right)_{r-|\theta|}}{\left(q v_{i} / q^{r-|\theta|} u_{k} ; q\right)_{r-|\theta|}} \frac{\left(q u_{i} / t q^{r-|\theta|} u_{k} ; q\right)_{r-|\theta|}}{\left(u_{i} / q^{r-|\theta|} u_{k} ; q\right)_{r-|\theta|}} \\
& \times \prod_{j=k+2}^{n+1} \frac{\left(t q^{r-|\theta|} u_{k} / v_{j} ; q\right)_{\theta_{j}}}{\left(q u^{r-|\theta|+1} u_{k} / v_{j} ; q\right)_{\theta_{j}}} \frac{\left(t^{2} v_{j} ; q\right)_{\theta_{j}}}{\left(u_{k} / t v_{j} ; q\right)_{\theta_{j}}} .
\end{aligned}
$$

We have obviously

$$
\frac{\left(q^{|\theta|-r+1} u_{i} / t u_{k} ; q\right)_{\theta_{i}}}{\left(q^{|\theta|-r} u_{i} / u_{k} ; q\right)_{\theta_{i}}} \frac{\left(u_{i} / u_{k} ; q\right)_{\theta_{i}-r+|\theta|}}{\left(q u_{i} / t u_{k} ; q\right)_{\theta_{i}-r+|\theta|}}=\frac{\left(q u_{i} / t q^{r-|\theta|} u_{k} ; q\right)_{r-|\theta|}}{\left(u_{i} / q^{r-|\theta|} u_{k} ; q\right)_{r-|\theta|}} .
$$

Using the identities

$$
\frac{\left(a q^{-n} ; q\right)_{n}}{\left(b q^{-n} ; q\right)_{n}}=\frac{(q / a ; q)_{n}}{(q / b ; q)_{n}}(a / b)^{n}
$$




$$
\frac{(a ; q)_{n}}{(b ; q)_{n}} \frac{(b ; q)_{n-k}}{(a ; q)_{n-k}}=\frac{\left(q^{1-n} / a ; q\right)_{k}}{\left(q^{1-n} / b ; q\right)_{k}}(a / b)^{k},
$$

we get

$$
\begin{aligned}
\frac{\left(t u_{i} / u_{k} ; q\right)_{\theta_{i}}}{\left(q u_{i} / u_{k} ; q\right)_{\theta_{i}}} \frac{\left(q u_{i} / u_{k} ; q\right)_{\theta_{i}-r+|\theta|}}{\left(t u_{i} / u_{k} ; q\right)_{\theta_{i}-r+|\theta|}} & =\frac{\left(q^{1-\theta_{i}} u_{k} / t u_{i} ; q\right)_{r-|\theta|}}{\left(q^{-\theta_{i}} u_{k} / u_{i} ; q\right)_{r-|\theta|}}(t / q)^{r-|\theta|} \\
& =\frac{\left(t v_{i} / q^{r-|\theta|} u_{k} ; q\right)_{r-|\theta|}}{\left(q v_{i} / q^{r-|\theta|} u_{k} ; q\right)_{r-|\theta|}} .
\end{aligned}
$$

Similarly, we obtain

$$
\begin{aligned}
(t / q)^{\theta_{j}} \frac{\left(q^{|\theta|-r+1} u_{j} / t u_{k} ; q\right)_{\theta_{j}}}{\left(q^{|\theta|-r} u_{j} / u_{k} ; q\right)_{\theta_{j}}} & =\frac{\left(t q^{r-|\theta|} u_{k} / v_{j} ; q\right)_{\theta_{j}}}{\left(q^{r-|\theta|+1} u_{k} / v_{j} ; q\right)_{\theta_{j}}} \\
(q / t)^{\theta_{j}} \frac{\left(t^{2} u_{j} / u_{k} ; q\right)_{\theta_{j}}}{\left(q t u_{j} / u_{k} ; q\right)_{\theta_{j}}} & =\frac{\left(q u_{k} / t^{2} v_{j} ; q\right)_{\theta_{j}}}{\left(u_{k} / t v_{j} ; q\right)_{\theta_{j}}}
\end{aligned}
$$

Finally, we have proved the following Pieri formula.

Theorem 3.2 Let $\lambda=\sum_{i=1}^{n} \lambda_{i} \omega_{i}$ be a dominant weight, and $r \in \mathbb{N}$. Assume $\lambda_{k}=0$ for some fixed $1 \leq k \leq n$. Define

$$
u_{i}= \begin{cases}q^{-r} t^{-2}, & i=0, \\ q^{-r+\sum_{j=i}^{k-1} \lambda_{j}} t^{k-i-1}, & 1 \leq i \leq k-1, \\ q^{-r-\sum_{j=k+1}^{i+1} \lambda_{j}} t^{k-i-3}, & k \leq i \leq n-1 .\end{cases}
$$

We have

$$
P_{r \omega_{1}} P_{\lambda}=\sum_{\substack{\theta=\left(\theta_{1}, \ldots, \theta_{n-1}\right) \in \mathbb{N}^{n-1} \\|\theta| \leq r}} D_{\theta}\left(u_{0}, u_{1}, \ldots, u_{n-1} ; k, r\right) P_{\lambda+\rho}
$$

with

$$
\begin{aligned}
\rho= & \sum_{i=1}^{k-2}\left(\theta_{i}-\theta_{i+1}\right) \omega_{i}+\theta_{k-1} \omega_{k-1}+(r-|\theta|)\left(\omega_{k}-\omega_{k-1}\right)-\theta_{k} \omega_{k+1} \\
& +\sum_{i=k+2}^{n}\left(\theta_{i-2}-\theta_{i-1}\right) \omega_{i} .
\end{aligned}
$$

Remark For $k=1,2$ (resp. $k=n, n-1$ ), the first (resp. the last) sum in the above expression of $\rho$ must be understood as zero. This convention will be kept in the next sections. 


\section{A recurrence formula}

Given two multiintegers $\beta=\left(\beta_{1}, \ldots, \beta_{n-1}\right)$ and $\kappa=\left(\kappa_{1}, \ldots, \kappa_{n-1}\right) \in \mathbb{Z}^{n-1}$, we write $\beta \geq \kappa$ for $\beta_{i} \geq \kappa_{i}(1 \leq i \leq n-1)$. We say that an infinite $(n-1)$-dimensional matrix $F=\left(f_{\beta \kappa}\right)_{\beta, \kappa \in \mathbb{Z}^{n-1}}$ is lower-triangular if $f_{\beta \kappa}=0$ unless $\beta \geq \kappa$. When all $f_{\kappa \kappa} \neq 0$, there exists a unique lower-triangular matrix $G=\left(g_{\kappa \gamma}\right)_{\kappa, \gamma \in \mathbb{Z}^{n-1}}$ such that

$$
\sum_{\beta \geq \kappa \geq \gamma} f_{\beta \kappa} g_{\kappa \gamma}=\delta_{\beta \gamma}
$$

for all $\beta, \gamma \in \mathbb{Z}^{n-1}$, where $\delta_{\beta \gamma}$ is the usual Kronecker symbol. We refer to $F$ and $G$ as mutually inverse.

Such a pair of infinite multidimensional inverse matrices is given in the Appendix, as a corollary of [3, Theorem 2.7] (and, in fact, equivalent to the latter). This result is essential for our purpose.

Given $n$ indeterminates $\left(u_{0}, u_{1}, \ldots, u_{n-1}\right), \theta=\left(\theta_{1}, \ldots, \theta_{n-1}\right) \in \mathbb{N}^{n-1}$, and $k, r \in \mathbb{N}$ with $1 \leq k \leq n$, we define

$$
\begin{aligned}
C_{\theta_{1}, \ldots, \theta_{n-1}}\left(u_{0}, u_{1}, \ldots, u_{n-1} ; k, r\right) \\
=q^{|\theta|} \frac{\left(t^{2} u_{0} ; q\right)_{|\theta|}}{\left(q t u_{0} ; q\right)_{|\theta|}} \prod_{i=1}^{n-1} \frac{(q / t ; q)_{\theta_{i}}}{(q ; q)_{\theta_{i}}} \frac{\left(q u_{i} ; q\right)_{\theta_{i}}}{\left(q t u_{i} ; q\right)_{\theta_{i}}} \\
\quad \times \prod_{1 \leq i<j \leq n-1} \frac{\left(q v_{i} / t v_{j} ; q\right)_{\theta_{j}}}{\left(q v_{i} / v_{j} ; q\right)_{\theta_{j}}} \frac{\left(t u_{i} / v_{j} ; q\right)_{\theta_{j}}}{\left(u_{i} / v_{j} ; q\right)_{\theta_{j}}} \\
\quad \times \prod_{i=1}^{k-1} \frac{\left(u_{i} / t u_{0} ; q\right)_{\theta_{i}}}{\left(q u_{i} / t^{2} u_{0} ; q\right)_{\theta_{i}}} \frac{\left(q t u_{0} / u_{i} ; q\right)_{r}}{\left(t^{2} u_{0} / u_{i} ; q\right)_{r}} \frac{\left(t u_{0} / u_{i} ; q\right)_{r}}{\left(q u_{0} / u_{i} ; q\right)_{r}} \prod_{i=k}^{n-1} \frac{\left(t u_{i} / u_{0} ; q\right)_{\theta_{i}}}{\left(q u_{i} / u_{0} ; q\right)_{\theta_{i}}} \\
\quad \times \frac{1}{\Delta(v)} \operatorname{det}_{1 \leq i, j \leq n-1}\left[v_{i}^{n-j-1}\left(1-t^{j-1} \frac{1-t v_{i}}{1-v_{i}} \prod_{s=1}^{n-1} \frac{v_{i}-u_{s}}{v_{i}-t u_{s}}\right)\right]
\end{aligned}
$$

with $\Delta(v)$ the Vandermonde determinant $\prod_{1 \leq i<j \leq n-1}\left(v_{i}-v_{j}\right)$. Here is our main result.

Theorem 4.1 Let $\lambda=\sum_{i=1}^{n} \lambda_{i} \omega_{i}$ be a dominant weight. Assume $\lambda_{k}=0$ for some fixed $1 \leq k \leq n$. For any positive integer $r \leq \lambda_{k-1}$, the weight

$$
\lambda^{(r)}=\lambda+r\left(\omega_{k}-\omega_{k-1}\right)=\lambda+r \varepsilon_{k}
$$

is dominant. Define

$$
u_{i}= \begin{cases}q^{-r} t^{-2}, & i=0, \\ q^{-r+\sum_{j=i}^{k-1} \lambda_{j}} t^{k-i-1}, & 1 \leq i \leq k-1, \\ q^{-r-\sum_{j=k+1}^{i+1} \lambda_{j}} t^{k-i-3}, & k \leq i \leq n-1 .\end{cases}
$$


We have

$$
P_{\lambda^{(r)}}=\sum_{\substack{\theta=\left(\theta_{1}, \ldots, \theta_{n-1}\right) \in \mathbb{N}^{n-1} \\|\theta| \leq r}} C_{\theta}\left(u_{0}, u_{1}, \ldots, u_{n-1} ; k, r\right) P_{(r-|\theta|) \omega_{1}} P_{\lambda+\rho}
$$

with

$$
\rho=\sum_{i=1}^{k-2}\left(\theta_{i}-\theta_{i+1}\right) \omega_{i}+\theta_{k-1} \omega_{k-1}-\theta_{k} \omega_{k+1}+\sum_{i=k+2}^{n}\left(\theta_{i-2}-\theta_{i-1}\right) \omega_{i} .
$$

Remark The weight $\lambda+\rho$ has no component on $\omega_{k}$. Further, similarly as in Theorem 3.1 (see the remark following the proof of that theorem), the condition $\lambda+\rho \in P^{+}$is necessarily satisfied in Theorem 4.2 as soon as $C_{\theta}\left(u_{0}, u_{1}, \ldots, u_{n-1}\right.$; $k, r) \neq 0$. We omit the details which involve a tedious case-by-case analysis.

Proof We make use of the multidimensional matrix inverse given in the Appendix. Let $\beta=\left(\beta_{1}, \ldots, \beta_{n-1}\right), \kappa=\left(\kappa_{1}, \ldots, \kappa_{n-1}\right), \gamma=\left(\gamma_{1}, \ldots, \gamma_{n-1}\right) \in \mathbb{Z}^{n-1}$. If we define

$$
\begin{aligned}
& f_{\beta \kappa}=C_{\beta_{1}-\kappa_{1}, \ldots, \beta_{n-1}-\kappa_{n-1}}\left(q^{|\kappa|} u_{0}, q^{\kappa_{1}+|\kappa|} u_{1}, \ldots, q^{\kappa_{n-1}+|\kappa|} u_{n-1} ; k, r-|\kappa|\right), \\
& g_{\kappa \gamma}=D_{\kappa_{1}-\gamma_{1}, \ldots, \kappa_{n-1}-\gamma_{n-1}}\left(q^{|\gamma|} u_{0}, q^{\gamma_{1}+|\gamma|} u_{1}, \ldots, q^{\gamma_{n-1}+|\gamma|} u_{n-1} ; k, r-|\gamma|\right),
\end{aligned}
$$

by this result, the infinite lower-triangular multidimensional matrices $\left(f_{\beta \kappa}\right)_{\beta, \kappa \in \mathbb{Z}^{n-1}}$ and $\left(g_{\kappa \gamma}\right)_{\kappa, \gamma \in \mathbb{Z}^{n-1}}$ are mutually inverse.

Now let us replace $\lambda_{i}$ in Theorem 3.2 by $\lambda_{i}+\gamma_{i}-\gamma_{i+1}$ for $1 \leq i \leq k-2, \lambda_{k-1}$ by $\lambda_{k-1}+\gamma_{k-1}, \lambda_{k+1}$ by $\lambda_{k+1}-\gamma_{k}, \lambda_{i}$ by $\lambda_{i}+\gamma_{i-2}-\gamma_{i-1}$ for $k+2 \leq i \leq n$, and $r$ by $r-|\gamma|$. Then $u_{0}$ is replaced by $q^{|\gamma|} u_{0}$, and $u_{i}$ by $q^{\gamma_{i}+|\gamma|} u_{i}$ for $1 \leq i \leq n-1$. In explicit terms, we are considering the identity

$$
\begin{aligned}
& P_{(r-|\gamma|) \omega_{1}} P_{\lambda+\tilde{\gamma}} \sum_{\substack{\theta=\left(\theta_{1}, \ldots, \theta_{n-1}\right) \in \mathbb{N}^{n-1} \\
|\theta| \leq r}} D_{\theta}\left(q^{|\gamma|} u_{0}, q^{\gamma_{1}+|\gamma|} u_{1}, \ldots, q^{\gamma_{n-1}+|\gamma|} u_{n-1} ; k, r-|\gamma|\right) P_{\lambda+\tilde{\gamma}+\rho}
\end{aligned}
$$

with

$$
u_{i}= \begin{cases}q^{-r} t^{-2}, & i=0, \\ q^{-r+\sum_{j=i}^{k-1} \lambda_{j}} t^{k-i-1}, & 1 \leq i \leq k-1, \\ q^{-r-\sum_{j=k+1}^{i+1} \lambda_{j}} t^{k-i-3}, & k \leq i \leq n-1,\end{cases}
$$

and

$$
\begin{aligned}
\rho= & \sum_{i=1}^{k-2}\left(\theta_{i}-\theta_{i+1}\right) \omega_{i}+\theta_{k-1} \omega_{k-1}+(r-|\theta|)\left(\omega_{k}-\omega_{k-1}\right)-\theta_{k} \omega_{k+1} \\
& +\sum_{i=k+2}^{n}\left(\theta_{i-2}-\theta_{i-1}\right) \omega_{i},
\end{aligned}
$$




$$
\tilde{\gamma}=\sum_{i=1}^{k-2}\left(\gamma_{i}-\gamma_{i+1}\right) \omega_{i}+\gamma_{k-1} \omega_{k-1}-\gamma_{k} \omega_{k+1}+\sum_{i=k+2}^{n}\left(\gamma_{i-2}-\gamma_{i-1}\right) \omega_{i}
$$

After substituting the summation indices $\theta_{i} \mapsto \kappa_{i}-\gamma_{i}$ for $1 \leq i \leq n-1$, we obtain exactly

$$
\sum_{\kappa \in \mathbb{Z}^{n-1}} g_{\kappa \gamma} y_{\kappa}=w_{\gamma} \quad\left(\gamma \in \mathbb{Z}^{n-1}\right)
$$

with

$$
y_{\kappa}=P_{\lambda+\tilde{\kappa}}, \quad w_{\gamma}=P_{(r-|\gamma|) \omega_{1}} P_{\lambda+\tilde{\gamma}}
$$

and

$$
\begin{aligned}
\tilde{\kappa}= & \sum_{i=1}^{k-2}\left(\kappa_{i}-\kappa_{i+1}\right) \omega_{i}+\kappa_{k-1} \omega_{k-1}+(r-|\kappa|)\left(\omega_{k}-\omega_{k-1}\right)-\kappa_{k} \omega_{k+1} \\
& +\sum_{i=k+2}^{n}\left(\kappa_{i-2}-\kappa_{i-1}\right) \omega_{i} .
\end{aligned}
$$

This immediately yields the inverse relation

$$
\sum_{\beta \in \mathbb{Z}^{n-1}} f_{\beta \kappa} w_{\beta}=y_{\kappa} \quad\left(\kappa \in \mathbb{Z}^{n-1}\right) .
$$

We conclude by setting $\kappa_{i}=0$ for all $1 \leq i \leq n-1$.

Finally, by the substitutions $r \rightarrow \lambda_{k}$ and $\lambda_{k-1} \rightarrow \lambda_{k-1}+\lambda_{k}$, we obtain the following very remarkable expansion.

Theorem 4.2 Let $\lambda=\sum_{i=1}^{n} \lambda_{i} \omega_{i}$ be a dominant weight, and $k \in \mathbb{N}$ fixed with $1 \leq$ $k \leq n$. Define

$$
u_{i}= \begin{cases}q^{-\lambda_{k}} t^{-2}, & i=0, \\ q^{\sum_{j=i}^{k-1} \lambda_{j}} t^{k-i-1}, & 1 \leq i \leq k-1, \\ q^{-\sum_{j=k}^{i+1} \lambda_{j}} t^{k-i-3}, & k \leq i \leq n-1,\end{cases}
$$

and $\mu=\lambda-\lambda_{k}\left(\omega_{k}-\omega_{k-1}\right)=\lambda-\lambda_{k} \varepsilon_{k}$. We have

$$
P_{\lambda}=\sum_{\substack{\theta=\left(\theta_{1}, \ldots, \theta_{n-1}\right) \in \mathbb{N}^{n-1} \\|\theta| \leq \lambda_{k}}} C_{\theta}\left(u_{0}, u_{1}, \ldots, u_{n-1} ; k, \lambda_{k}\right) P_{\left(\lambda_{k}-|\theta|\right) \omega_{1}} P_{\mu+\rho}
$$

with

$$
\rho=\sum_{i=1}^{k-2}\left(\theta_{i}-\theta_{i+1}\right) \omega_{i}+\theta_{k-1} \omega_{k-1}-\theta_{k} \omega_{k+1}+\sum_{i=k+2}^{n}\left(\theta_{i-2}-\theta_{i-1}\right) \omega_{i} .
$$


Remark Observe that the weights $\mu$ and $\mu+\rho$ have no component on $\omega_{k}$.

The special case $k=n$ is worth writing out explicitly.

Corollary Let $\lambda=\sum_{i=1}^{n} \lambda_{i} \omega_{i}$ be a dominant weight. Define $u_{0}=q^{-\lambda_{n}} t^{-2}$ and $u_{i}=$ $q^{\sum_{l=i}^{n-1} \lambda_{l}} t^{n-i-1}(1 \leq i \leq n-1)$. We have

$$
P_{\lambda}=\sum_{\substack{\theta=\left(\theta_{1}, \ldots, \theta_{n-1}\right) \in \mathbb{N}^{n-1} \\|\theta| \leq \lambda_{n}}} C_{\theta}\left(u_{0}, u_{1}, \ldots, u_{n-1} ; n, \lambda_{n}\right) P_{\left(\lambda_{n}-|\theta|\right) \omega_{1}} P_{\mu}
$$

with $\mu=\sum_{i=1}^{n-2}\left(\lambda_{i}+\theta_{i}-\theta_{i+1}\right) \omega_{i}+\left(\lambda_{n-1}+\lambda_{n}+\theta_{n-1}\right) \omega_{n-1}$.

The reader may check that this is exactly Theorem 4.1 of [3] (with $n \mapsto n-1$ ), written for $x_{1} \cdots x_{n+1}=1$, up to the normalization $Q_{\lambda}=b_{\lambda} P_{\lambda}$ with

$$
b_{\lambda}=\prod_{1 \leq i \leq j \leq n} \frac{\left(q^{\sum_{l=i}^{j-1} \lambda_{l}} t^{j-i+1} ; q\right) \lambda_{j}}{\left(q^{1+\sum_{l=i}^{j-1} \lambda_{l}} t^{j-i} ; q\right)_{\lambda_{j}}}=\prod_{1 \leq i \leq j \leq n} \frac{\left(t u_{i} / u_{j} ; q\right) \lambda_{j}}{\left(q u_{i} / u_{j} ; q\right)_{\lambda_{j}}},
$$

where we set $u_{n}=1 / t$.

\section{Examples}

In this section we write out the formulas in Theorem 4.2 explicitly for $n=2,3$.

\subsection{The root system $A_{2}$}

For $k=2$, we have $u_{0}=q^{-\lambda_{2}} / t^{2}, u_{1}=q^{\lambda_{1}}$, and

$$
\begin{aligned}
C_{\theta}\left(u_{0}, u_{1} ; 2, r\right)= & q^{\theta} \frac{\left(t^{2} u_{0} ; q\right)_{\theta}}{\left(q t u_{0} ; q\right)_{\theta}} \frac{(q / t ; q)_{\theta}}{(q ; q)_{\theta}} \frac{\left(q u_{1} ; q\right)_{\theta}}{\left(q t u_{1} ; q\right)_{\theta}} \frac{\left(u_{1} / t u_{0} ; q\right)_{\theta}}{\left(q u_{1} / t^{2} u_{0} ; q\right)_{\theta}} \\
& \times \frac{\left(q t u_{0} / u_{1} ; q\right)_{r}}{\left(t u_{0} u_{0} / u_{1} ; q\right)_{r}} \frac{\left(u_{1} ; q\right)_{r}}{\left(q u_{0} / u_{1} ; q\right)_{r}}\left(1-\frac{1-t v_{1}}{1-v_{1}} \frac{v_{1}-u_{1}}{v_{1}-t u_{1}}\right) .
\end{aligned}
$$

After some simplifications, we obtain

$$
P_{\lambda_{1} \omega_{1}+\lambda_{2} \omega_{2}}=\sum_{\theta \in \mathbb{N}} C_{\theta}^{(2)}(\lambda) P_{\left(\lambda_{2}-\theta\right) \omega_{1}} P_{\left(\lambda_{1}+\lambda_{2}+\theta\right) \omega_{1}}
$$

with

$$
\begin{aligned}
C_{\theta}^{(2)}(\lambda) & =C_{\theta}\left(u_{0}, u_{1} ; 2, \lambda_{2}\right) \\
& =t^{\theta} \frac{\left(q^{\lambda_{2}-\theta+1} ; q\right)_{\theta}}{\left(t q^{\lambda_{2}-\theta} ; q\right)_{\theta}} \frac{(1 / t ; q)_{\theta}}{(q ; q)_{\theta}} \frac{\left(q^{\lambda_{1}+1} ; q\right)_{\theta}}{\left(t q^{\lambda_{1}+1} ; q\right)_{\theta}}
\end{aligned}
$$




$$
\times \frac{\left(t q^{\lambda_{1}} ; q\right)_{\lambda_{2}+\theta}}{\left(q^{\lambda_{1}+1} ; q\right)_{\lambda_{2}+\theta}} \frac{\left(t q^{\lambda_{1}+1} ; q\right)_{\lambda_{2}}}{\left(t^{2} q^{\lambda_{1}} ; q\right)_{\lambda_{2}}} \frac{1-q^{\lambda_{1}+2 \theta}}{1-q^{\lambda_{1}+\theta}} .
$$

This result may be compared with the Jing-Józefiak classical result [1], more precisely, with its restriction to three variables $\left(x_{1}, x_{2}, x_{3}\right)$ subject to $x_{1} x_{2} x_{3}=1$. Namely, given a partition $\left(\mu_{1}, \mu_{2}\right)$, the Macdonald symmetric function $\mathcal{P}_{\left(\mu_{1}, \mu_{2}\right)}(q, t)$ is given by

$$
\mathcal{P}_{\left(\mu_{1}, \mu_{2}\right)}=\sum_{\theta \in \mathbb{N}} \mathcal{C}_{\theta}(\mu) \mathcal{P}_{\left(\mu_{2}-\theta\right)} \mathcal{P}_{\left(\mu_{1}+\theta\right)}
$$

with

$$
\begin{aligned}
\mathcal{C}_{\theta}(\mu)= & \frac{\left(t q^{\mu_{1}-\mu_{2}+1} ; q\right)_{\mu_{2}}}{\left(t^{2} q^{\mu_{1}-\mu_{2}} ; q\right)_{\mu_{2}}} \frac{\left(q^{\mu_{2}-\theta+1} ; q\right)_{\theta}}{\left(t q^{\mu_{2}-\theta} ; q\right)_{\theta}} \frac{\left(t q^{\mu_{1}-\mu_{2}} ; q\right)_{\mu_{2}+\theta}}{\left(q^{\mu_{1}-\mu_{2}+1} ; q\right)_{\mu_{2}+\theta}} \\
& \times t^{\theta} \frac{(1 / t ; q)_{\theta}}{(q ; q)_{\theta}} \frac{\left(q^{\mu_{1}-\mu_{2}+1} ; q\right)_{\theta}}{\left(t q^{\mu_{1}-\mu_{2}+1} ; q\right)_{\theta}} \frac{1-q^{\mu_{1}-\mu_{2}+2 \theta}}{1-q^{\mu_{1}-\mu_{2}+\theta}} .
\end{aligned}
$$

Our formula is equivalent to the main result of [1] by the correspondence $\lambda_{1}=\mu_{1}-$ $\mu_{2}, \lambda_{2}=\mu_{2}$ between dominant weights and partitions, recalled in Sect. 2.

For $k=1$, we have $u_{0}=q^{-\lambda_{1}} / t^{2}, u_{1}=q^{-\lambda_{1}-\lambda_{2}} / t^{3}$, and

$$
\begin{aligned}
C_{\theta}\left(u_{0}, u_{1} ; 1, r\right)= & q^{\theta} \frac{\left(t^{2} u_{0} ; q\right)_{\theta}}{\left(q t u_{0} ; q\right)_{\theta}} \frac{(q / t ; q)_{\theta}}{(q ; q)_{\theta}} \frac{\left(q u_{1} ; q\right)_{\theta}}{\left(q t u_{1} ; q\right)_{\theta}} \frac{\left(t u_{1} / u_{0} ; q\right)_{\theta}}{\left(q u_{1} / u_{0} ; q\right)_{\theta}} \\
& \times\left(1-\frac{1-t v_{1}}{1-v_{1}} \frac{v_{1}-u_{1}}{v_{1}-t u_{1}}\right) .
\end{aligned}
$$

After some simplifications, we obtain

$$
P_{\lambda_{1} \omega_{1}+\lambda_{2} \omega_{2}}=\sum_{\theta \in \mathbb{N}} C_{\theta}^{(1)}(\lambda) P_{\left(\lambda_{1}-\theta\right) \omega_{1}} P_{\left(\lambda_{2}-\theta\right) \omega_{2}}
$$

with

$$
\begin{aligned}
C_{\theta}^{(1)}(\lambda)= & C_{\theta}\left(u_{0}, u_{1} ; 1, \lambda_{1}\right) \\
= & t^{\theta} \frac{(1 / t ; q)_{\theta}}{(q ; q)_{\theta}} \frac{\left(q^{\lambda_{1}} ; 1 / q\right)_{\theta}}{\left(t q^{\lambda_{1}-1} ; 1 / q\right)_{\theta}} \frac{\left(q^{\lambda_{2}} ; 1 / q\right)_{\theta}}{\left(t q^{\lambda_{2}-1} ; 1 / q\right)_{\theta}} \frac{\left(t^{3} q^{\lambda_{1}+\lambda_{2}-1} ; 1 / q\right)_{\theta}}{\left(t^{2} q^{\lambda_{1}+\lambda_{2}-1} ; 1 / q\right)_{\theta}} \\
& \times \frac{1-t^{3} q^{\lambda_{1}+\lambda_{2}-2 \theta}}{1-t^{3} q^{\lambda_{1}+\lambda_{2}-\theta}} .
\end{aligned}
$$

We thus recover exactly the result of Perelomov, Ragoucy, and Zaugg [9, Theorem 1(a)].

\subsection{The root system $A_{3}$}

For $k=1,2,3$, our formulas in Theorem 4.2 write respectively as

$$
P_{\lambda_{1} \omega_{1}+\lambda_{2} \omega_{2}+\lambda_{3} \omega_{3}}=\sum_{(i, j) \in \mathbb{N}^{2}} C_{i j}^{(1)}(\lambda) P_{\left(\lambda_{1}-i-j\right) \omega_{1}} P_{\left(\lambda_{2}-i\right) \omega_{2}+\left(\lambda_{3}+i-j\right) \omega_{3}}
$$




$$
\begin{aligned}
& =\sum_{(i, j) \in \mathbb{N}^{2}} C_{i j}^{(2)}(\lambda) P_{\left(\lambda_{2}-i-j\right) \omega_{1}} P_{\left(\lambda_{1}+\lambda_{2}+i\right) \omega_{1}+\left(\lambda_{3}-j\right) \omega_{3}} \\
& =\sum_{(i, j) \in \mathbb{N}^{2}} C_{i j}^{(3)}(\lambda) P_{\left(\lambda_{3}-i-j\right) \omega_{1}} P_{\left(\lambda_{1}+i-j\right) \omega_{1}+\left(\lambda_{2}+\lambda_{3}+j\right) \omega_{2}} .
\end{aligned}
$$

In order to make these expansions explicit, we need to evaluate the determinant of the 2 by 2 matrix $A$ given by

$$
A_{k l}=v_{k}^{2-l}\left(1-t^{l-1} \frac{1-t v_{k}}{1-v_{k}} \frac{v_{k}-u_{1}}{v_{k}-t u_{1}} \frac{v_{k}-u_{2}}{v_{k}-t u_{2}}\right)
$$

with $v_{1}=q^{i} u_{1}, v_{2}=q^{j} u_{2}$.

More precisely, we need to compute the quotient of this determinant by the Vandermonde determinant $v_{1}-v_{2}=q^{i} u_{1}-q^{j} u_{2}$. There is no evidence that this quotient may be written in canonical form. Inspired by the explicit result of [2, Theorem 1] (see below), we write this quotient of determinants as

$$
\begin{aligned}
\frac{\operatorname{det} A}{q^{i} u_{1}-q^{j} u_{2}}= & \frac{(t-1)^{2}}{\left(t-q^{i}\right)\left(t-q^{j}\right)} \\
& \times\left(\frac{1-q^{2 i} u_{1}}{1-q^{i} u_{1}} \frac{1-q^{2 j} u_{2}}{1-q^{j} u_{2}}\left(1+t^{-1} \frac{1-q^{i}}{1-q^{i} u_{1} / t u_{2}} \frac{1-q^{j}}{1-q^{j} u_{2} / t u_{1}}\right)\right. \\
& \left.-\left(q^{i} u_{1}+q^{j} u_{2}\right) \frac{1-q^{i}}{1-q^{i} u_{1}} \frac{1-q^{j}}{1-q^{j} u_{2}} \frac{1-q^{i} / t}{1-q^{i} u_{1} / t u_{2}} \frac{1-q^{j} / t}{1-q^{j} u_{2} / t u_{1}}\right) .
\end{aligned}
$$

The above identity (which is not trivial) may be easily verified by using any formal calculus software.

Next, for $(i, j) \in \mathbb{N}^{2}$, we define

$$
\begin{aligned}
\nabla_{i j}( & \left.u_{0}, u_{1}, u_{2}\right) \\
= & q^{i+j} \frac{\left(t^{2} u_{0} ; q\right)_{i+j}}{\left(q t u_{0} ; q\right)_{i+j}} \frac{(1 / t ; q)_{i}}{(q ; q)_{i}} \frac{\left(u_{1} ; q\right)_{i}}{\left(q t u_{1} ; q\right)_{i}} \frac{(1 / t ; q)_{j}}{(q ; q)_{j}} \\
& \times \frac{\left(u_{2} ; q\right)_{j}}{\left(q t u_{2} ; q\right)_{j}} \frac{\left(q^{i-j+1} u_{1} / t u_{2} ; q\right)_{j}}{\left(q^{i-j+1} u_{1} / u_{2} ; q\right)_{j}} \frac{\left(t q^{-j} u_{1} / u_{2} ; q\right)_{j}}{\left(q^{-j} u_{1} / u_{2} ; q\right)_{j}} \\
& \times\left(\frac{1-q^{2 i} u_{1}}{1-u_{1}} \frac{1-q^{2 j} u_{2}}{1-u_{2}}\left(1+t^{-1} \frac{1-q^{i}}{1-q^{i} u_{1} / t u_{2}} \frac{1-q^{j}}{1-q^{j} u_{2} / t u_{1}}\right)\right. \\
& \left.-\left(q^{i} u_{1}+q^{j} u_{2}\right) \frac{1-q^{i}}{1-u_{1}} \frac{1-q^{j}}{1-u_{2}} \frac{1-q^{i} / t}{1-q^{i} u_{1} / t u_{2}} \frac{1-q^{j} / t}{1-q^{j} u_{2} / t u_{1}}\right) .
\end{aligned}
$$

It is readily verified that we have

$$
\frac{C_{i j}\left(u_{0}, u_{1}, u_{2} ; 1, r\right)}{\nabla_{i j}\left(u_{0}, u_{1}, u_{2}\right)}=\frac{\left(t u_{1} / u_{0} ; q\right)_{i}}{\left(q u_{1} / u_{0} ; q\right)_{i}} \frac{\left(t u_{2} / u_{0} ; q\right)_{j}}{\left(q u_{2} / u_{0} ; q\right)_{j}},
$$




$$
\begin{aligned}
\frac{C_{i j}\left(u_{0}, u_{1}, u_{2} ; 2, r\right)}{\nabla_{i j}\left(u_{0}, u_{1}, u_{2}\right)}= & \frac{\left(u_{1} / t u_{0} ; q\right)_{i}}{\left(q u_{1} / t^{2} u_{0} ; q\right)_{i}} \frac{\left(q t u_{0} / u_{1} ; q\right)_{r}}{\left(t^{2} u_{0} / u_{1} ; q\right)_{r}} \frac{\left(t u_{0} / u_{1} ; q\right)_{r}}{\left(q u_{0} / u_{1} ; q\right)_{r}} \frac{\left(t u_{2} / u_{0} ; q\right)_{j}}{\left(q u_{2} / u_{0} ; q\right)_{j}}, \\
\frac{C_{i j}\left(u_{0}, u_{1}, u_{2} ; 3, r\right)}{\nabla_{i j}\left(u_{0}, u_{1}, u_{2}\right)}= & \frac{\left(u_{1} / t u_{0} ; q\right)_{i}}{\left(q u_{1} / t^{2} u_{0} ; q\right)_{i}} \frac{\left(q t u_{0} / u_{1} ; q\right)_{r}}{\left(t u_{0} / u_{1} ; q\right)_{r}} \frac{\left(t^{2} u_{0} / u_{1} ; q\right)_{r}}{\left(q u_{0} / u_{1} ; q\right)_{r}} \\
& \times \frac{\left(u_{2} / t u_{0} ; q\right)_{j}}{\left(q u_{2} / t^{2} u_{0} ; q\right)_{j}} \frac{\left(q t u_{0} / u_{2} ; q\right)_{r}}{\left(t u_{0} u_{0} / u_{2} ; q\right)_{r}} \frac{\left(t u_{2} ; q\right)_{r}}{\left(q u_{0} / u_{2} ; q\right)_{r}}
\end{aligned}
$$

Now, by Theorem 4.2 the respective recurrence coefficients are determined to be

$$
\begin{aligned}
& C_{i j}^{(1)}(\lambda)=C_{i j}\left(q^{-\lambda_{1}} / t^{2}, q^{-\lambda_{1}-\lambda_{2}} / t^{3}, q^{-\lambda_{1}-\lambda_{2}-\lambda_{3}} / t^{4} ; 1, \lambda_{1}\right), \\
& C_{i j}^{(2)}(\lambda)=C_{i j}\left(q^{-\lambda_{2}} / t^{2}, q^{\lambda_{1}}, q^{-\lambda_{2}-\lambda_{3}} / t^{3} ; 2, \lambda_{2}\right), \\
& C_{i j}^{(3)}(\lambda)=C_{i j}\left(q^{-\lambda_{3}} / t^{2}, q^{\lambda_{1}+\lambda_{2}} t, q^{\lambda_{2}} ; 3, \lambda_{3}\right) .
\end{aligned}
$$

The cases $k=1,2$ are new. For $k=3$, we recover the first author's earlier result in [2, Theorem 1], more precisely, the restriction of this result to four variables $\left(x_{1}, x_{2}, x_{3}, x_{4}\right)$ subject to $x_{1} x_{2} x_{3} x_{4}=1$. Namely, given a partition $\left(\mu_{1}, \mu_{2}, \mu_{3}\right)$ and $u=q^{\mu_{1}-\mu_{2}}, v=q^{\mu_{2}-\mu_{3}}$, the Macdonald symmetric function $\mathcal{P}_{\left(\mu_{1}, \mu_{2}, \mu_{3}\right)}(q, t)$ is given by

$$
\mathcal{P}_{\left(\mu_{1}, \mu_{2}, \mu_{3}\right)}=\sum_{(i, j) \in \mathbb{N}^{2}} \mathcal{C}_{i j}(\mu) \mathcal{P}_{\left(\mu_{3}-i-j\right)} \mathcal{P}_{\left(\mu_{1}+i, \mu_{2}+j\right)}
$$

with

$$
\begin{aligned}
\mathcal{C}_{i j}(\mu)= & t^{i+j} \frac{(1 / t ; q)_{i}}{(q ; q)_{i}} \frac{(1 / t ; q)_{j}}{(q ; q)_{j}} \frac{(t u v ; q)_{i}}{\left(q t^{2} u v ; q\right)_{i}} \frac{(v ; q)_{j}}{(q t v ; q)_{j}} \frac{\left(q^{-j} t^{2} u ; q\right)_{i}}{\left(q^{-j} t u ; q\right)_{i}} \frac{(q u ; q)_{i}}{(q t u ; q)_{i}} \\
& \times \frac{(t ; q)_{\mu_{1}-\mu_{2}+i-j}}{(q ; q)_{\mu_{1}-\mu_{2}+i-j}} \frac{(t ; q)_{\mu_{2}+j}}{(q ; q)_{\mu_{2}+j}} \frac{(t ; q)_{\mu_{3}-i-j}}{(q ; q)_{\mu_{3}-i-j}} \frac{(q ; q)_{\mu_{1}-\mu_{2}}}{(t ; q)_{\mu_{1}-\mu_{2}}} \\
& \times \frac{(q ; q)_{\mu_{2}-\mu_{3}}}{(t ; q)_{\mu_{2}-\mu_{3}}} \frac{(q ; q)_{\mu_{3}}}{(t ; q)_{\mu_{3}}} \frac{\left(q^{i-j} t^{2} u ; q\right)_{\mu_{2}+j}}{\left(q^{i-j+1} t u ; q\right)_{\mu_{2}+j}} \frac{(q t u ; q)_{\mu_{2}-\mu_{3}}}{\left(t^{2} u ; q\right)_{\mu_{2}-\mu_{3}}} \\
& \times \frac{\left(q t^{2} u v ; q\right)_{\mu_{3}}}{\left(t^{3} u v ; q\right)_{\mu_{3}}} \frac{(q t q)_{\mu_{3}}}{\left(t^{2} v ; q\right)_{\mu_{3}}} \frac{1-q^{2 i} t u v}{1-t u v} \frac{1-q^{2 j} v}{1-v} \\
& \times\left(1+u \frac{1-q^{i}}{1-q^{i} u} \frac{1-q^{-j}}{1-q^{-j} t^{2} u}\left(t-v\left(q^{i} t u+q^{j}\right) \frac{t-q^{i}}{1-q^{2 i} t u v} \frac{t-q^{j}}{1-q^{2 j} v}\right)\right) .
\end{aligned}
$$

The reader may check our formula is indeed equivalent to [2, Theorem 1] by using the correspondence $\lambda_{1}=\mu_{1}-\mu_{2}, \lambda_{2}=\mu_{2}-\mu_{3}, \lambda_{3}=\mu_{3}$ between dominant weights and partitions.

\section{Final remark}

The Macdonald polynomial $P_{\lambda}, \lambda=\sum_{i=1}^{n} \lambda_{i} \omega_{i}$, is in bijective correspondence with the symmetric function $\mathcal{P}_{\mu}\left(x_{1}, \ldots, x_{n+1}\right)$ with $\mu=\left(\mu_{1}, \ldots, \mu_{n}\right), \mu_{i}=\sum_{j=i}^{n} \lambda_{j}$, 
subject to the condition $x_{1} \cdots x_{n+1}=1$. Therefore the $n$ recurrence relations that we have obtained for $P_{\lambda}$ may be expressed in terms of $\mathcal{P}_{\mu}\left(x_{1}, \ldots, x_{n+1}\right)$, subject to $x_{1} \cdots x_{n+1}=1$.

One may wonder whether this restriction can be removed. Equivalently, given some fixed integer $1 \leq k \leq n$, is it possible to expand the symmetric function $\mathcal{P}_{\mu}$ in terms of products $\mathcal{P}_{(r)} \mathcal{P}_{\rho}$ for partitions $\rho=\left(\rho_{1}, \ldots, \rho_{n}\right)$ satisfying $\rho_{k}=\rho_{k+1}$ ?

Such a development has been obtained in [3] for $k=n$, in which case $\rho_{n}=\rho_{n+1}=$ 0 . However, this method cannot be used for other values of $k$.

Actually, the Pieri expansion of $\mathcal{P}_{(r)} \mathcal{P}_{\rho}$ involves symmetric functions $\mathcal{P}_{\sigma}$ with $\sigma-\rho$ a horizontal $r$-strip. Hence some of these partitions $\sigma$ have length $l(\sigma)=n+1$. The only exception occurs for $k=n$ since in that case $\rho_{n}=0$ entails $l(\sigma) \leq n$.

Therefore, except for $k=n$, the Pieri multiplication does not conserve the space generated by $\left\{\mathcal{P}_{\kappa}, l(\kappa) \leq n\right\}$, and it is not possible to define a Pieri matrix to invert.

This difficulty does not arise in the $A_{n}$ framework. Then the Pieri matrix can be defined, because the condition $x_{1} \cdots x_{n+1}=1$ and the property [5, (4.17), p. 325]

$$
\mathcal{P}_{\left(\sigma_{1}, \ldots, \sigma_{n+1}\right)}\left(x_{1}, \ldots, x_{n+1}\right)=\left(x_{1} \cdots x_{n+1}\right)^{\sigma_{n+1}} \mathcal{P}_{\left(\sigma_{1}-\sigma_{n+1}, \ldots, \sigma_{n}-\sigma_{n+1}, 0\right)}\left(x_{1}, \ldots, x_{n+1}\right)
$$

allow us to deal with partitions of length $n+1$.

Acknowledgements We thank the anonymous referees for helpful comments. The second author was partly supported by FWF Austrian Science Fund grants P17563-N13 and S9607.

\section{Appendix: A multidimensional matrix inverse}

The following result (equivalent to one previously given in [3]) is crucial to obtain the recursion formula in Sect. 4.

Lemma Let $t, u_{0}, u_{1}, \ldots, u_{n}$ be indeterminates, and $r, k \in \mathbb{N}$ with $1 \leq k \leq n+1$. Define

$$
\begin{aligned}
f_{\beta \kappa}= & q^{|\beta|-|\kappa|} \frac{\left(t^{2} u_{0} ; q\right)_{|\beta|}}{\left(q t u_{0} ; q\right)_{|\beta|}} \frac{\left(q t u_{0} ; q\right)_{|\kappa|}}{\left(t^{2} u_{0} ; q\right)_{|\kappa|}} \prod_{i=1}^{n} \frac{(q / t ; q)_{\beta_{i}-\kappa_{i}}}{(q ; q)_{\beta_{i}-\kappa_{i}}} \frac{\left(q^{\kappa_{i}+|\kappa|+1} u_{i} ; q\right)_{\beta_{i}-\kappa_{i}}}{\left(q^{\kappa_{i}+|\kappa|+1} t u_{i} ; q\right)_{\beta_{i}-\kappa_{i}}} \\
& \times \prod_{i=1}^{k-1} \frac{\left(u_{i} / t u_{0} ; q\right)_{\beta_{i}}}{\left(q u_{i} / t^{2} u_{0} ; q\right)_{\beta_{i}}} \frac{\left(q u_{i} / t u_{0} ; q\right)_{\kappa_{i}}}{\left(u_{i} / u_{0} ; q\right)_{\kappa_{i}}} \\
& \times \prod_{i=1}^{k-1} \frac{\left(u_{i} / u_{0} ; q\right)_{|\kappa|-r+\kappa_{i}}}{\left(q u_{i} / t u_{0} ; q\right)_{|\kappa|-r+\kappa_{i}}} \frac{\left(q u_{i} / t^{2} u_{0} ; q\right)_{|\kappa|-r+\kappa_{i}}}{\left(u_{i} / t u_{0} ; q\right)_{|\kappa|-r+\kappa_{i}}} \\
& \times \prod_{i=k}^{n} \frac{\left(t u_{i} / u_{0} ; q\right)_{\beta_{i}}}{\left(q u_{i} / u_{0} ; q\right)_{\beta_{i}}} \frac{\left(q u_{i} / u_{0} ; q\right)_{\kappa_{i}}}{\left(t u_{i} / u_{0} ; q\right)_{\kappa_{i}}} \\
& \times \prod_{1 \leq i<j \leq n} \frac{\left(q^{\beta_{i}-\beta_{j}+1} u_{i} / t u_{j} ; q\right)_{\beta_{j}-\kappa_{j}}}{\left(q^{\beta_{i}-\beta_{j}+1} u_{i} / u_{j} ; q\right)_{\beta_{j}-\kappa_{j}}} \frac{\left(q^{\kappa_{i}-\beta_{j}} t u_{i} / u_{j} ; q\right)_{\beta_{j}-\kappa_{j}}}{\left(q^{\kappa_{i}-\beta_{j}} u_{i} / u_{j} ; q\right)_{\beta_{j}-\kappa_{j}}} \frac{1}{q^{\beta_{i}} u_{i}-q^{\beta_{j}} u_{j}}
\end{aligned}
$$




$$
\times \operatorname{det}_{1 \leq i, j \leq n}\left[\left(q^{\beta_{i}} u_{i}\right)^{n-j}\left(1-t^{j-1} \frac{\left(1-q^{\beta_{i}+|\kappa|} t u_{i}\right)}{\left(1-q^{\beta_{i}+|\kappa|} u_{i}\right)} \prod_{s=1}^{n} \frac{\left(q^{\beta_{i}} u_{i}-q^{\kappa_{s}} u_{s}\right)}{\left(q^{\beta_{i}} u_{i}-q^{\kappa_{s}} t u_{s}\right)}\right)\right]
$$

and

$$
\begin{aligned}
g_{\kappa \gamma}= & \left(\frac{q}{t}\right)^{|\kappa|-|\gamma|} \frac{\left(t^{2} u_{0} ; q\right)_{|\kappa|}}{\left(q t u_{0} ; q\right)_{|\kappa|}} \frac{\left(q t u_{0} ; q\right)_{|\gamma|}}{\left(t^{2} u_{0} ; q\right)_{|\gamma|}} \prod_{i=1}^{n} \frac{(t ; q)_{\kappa_{i}-\gamma_{i}}}{(q ; q)_{\kappa_{i}-\gamma_{i}}} \frac{\left(q^{\gamma_{i}+|\kappa|+1} u_{i} ; q\right)_{\kappa_{i}-\gamma_{i}}}{\left(q^{\gamma_{i}+|\kappa|} t u_{i} ; q\right)_{\kappa_{i}-\gamma_{i}}} \\
& \times \prod_{i=1}^{k-1} \frac{\left(u_{i} / u_{0} ; q\right)_{\kappa_{i}}}{\left(q u_{i} / t u_{0} ; q\right)_{\kappa_{i}}} \frac{\left(q u_{i} / t^{2} u_{0} ; q\right)_{\gamma_{i}}}{\left(u_{i} / t u_{0} ; q\right)_{\gamma_{i}}} \\
& \times \prod_{i=1}^{k-1} \frac{\left(q u_{i} / t u_{0} ; q\right)_{|\kappa|-r+\kappa_{i}}}{\left(u_{i} / u_{0} ; q\right)_{|\kappa|-r+\kappa_{i}}} \frac{\left(u_{i} / t u_{0} ; q\right)_{|\kappa|-r+\kappa_{i}}}{\left(q u_{i} / t^{2} u_{0} ; q\right)_{|\kappa|-r+\kappa_{i}}} \\
& \times \prod_{i=k}^{n} \frac{\left(t u_{i} / u_{0} ; q\right)_{\kappa_{i}}}{\left(q u_{i} / u_{0} ; q\right)_{\kappa_{i}}} \frac{\left(q u_{i} / u_{0} ; q\right)_{\gamma_{i}}}{\left(t u_{i} / u_{0} ; q\right)_{\gamma_{i}}} \\
& \times \prod_{1 \leq i<j \leq n} \frac{\left(q^{\kappa_{i}-\kappa_{j}} t u_{i} / u_{j} ; q\right)_{\kappa_{j}-\gamma_{j}}}{\left(q^{\kappa_{i}-\kappa_{j}+1} u_{i} / u_{j} ; q\right)_{\kappa_{j}-\gamma_{j}}} \frac{\left(q^{\gamma_{i}-\kappa_{j}+1} u_{i} / t u_{j} ; q\right)_{\kappa_{j}-\gamma_{j}}}{\left(q^{\gamma_{i}-\kappa_{j}} u_{i} / u_{j} ; q\right)_{\kappa_{j}-\gamma_{j}}} .
\end{aligned}
$$

Then the infinite lower-triangular $n$-dimensional matrices $\left(f_{\beta \kappa}\right)_{\beta, \kappa \in \mathbf{Z}^{n}}$ and $\left(g_{\kappa \gamma}\right)_{\kappa, \gamma \in \mathbf{Z}^{n}}$ are mutually inverse.

Proof Given two nonzero sequences $\left(\xi_{\kappa}\right)$ and $\left(\zeta_{\kappa}\right)$ and a pair of matrices $\left(f_{\beta \kappa}\right)$ and $\left(g_{\kappa \gamma}\right)$ which are mutually inverse, it is easily checked (using the trivial relation $\left.\frac{\xi_{\beta}}{\xi_{\gamma}} \delta_{\beta \gamma}=\delta_{\beta \gamma}\right)$ that the matrices $\left(f_{\beta \kappa} \xi_{\beta} / \zeta_{\kappa}\right)$ and $\left(g_{\kappa \gamma} \zeta_{\kappa} / \xi_{\gamma}\right)$ are mutually inverse.

We choose

$$
\begin{aligned}
\xi_{\kappa}= & \left(\frac{q}{t}\right)^{|\kappa|} \frac{\left(t^{2} u_{0} ; q\right)_{|\kappa|}}{\left(q t u_{0} ; q\right)_{|\kappa|}} \prod_{i=1}^{k-1} \frac{\left(u_{i} / t u_{0} ; q\right)_{\kappa_{i}}}{\left(q u_{i} / t^{2} u_{0} ; q\right)_{\kappa_{i}}} \prod_{i=k}^{n} \frac{\left(t u_{i} / u_{0} ; q\right)_{\kappa_{i}}}{\left(q u_{i} / u_{0} ; q\right)_{\kappa_{i}}} \\
& \times \prod_{1 \leq i<j \leq n} \frac{\left(q u_{i} / u_{j} ; q\right)_{\kappa_{i}-\kappa_{j}}}{\left(t u_{i} / u_{j} ; q\right)_{\kappa_{i}-\kappa_{j}}} \frac{\left(u_{i} / u_{j} ; q\right)_{\kappa_{i}-\kappa_{j}}}{\left(q u_{i} / t u_{j} ; q\right)_{\kappa_{i}-\kappa_{j}}}, \\
\zeta_{\kappa}= & \left(\frac{q}{t}\right)^{|\kappa|} \frac{\left(t^{2} u_{0} ; q\right)_{|\kappa|}}{\left(q t u_{0} ; q\right)_{|\kappa|}} \prod_{i=1}^{k-1} \frac{\left(u_{i} / u_{0} ; q\right)_{\kappa_{i}}}{\left(q u_{i} / t u_{0} ; q\right)_{\kappa_{i}}} \\
& \times \prod_{i=1}^{k-1} \frac{\left(q u_{i} / t u_{0} ; q\right)_{|\kappa|-r+\kappa_{i}}}{\left(u_{i} / u_{0} ; q\right)_{|\kappa|-r+\kappa_{i}}} \frac{\left(u_{i} / t u_{0} ; q\right)_{|\kappa|-r+\kappa_{i}}}{\left(q u_{i} / t^{2} u_{0} ; q\right)_{|\kappa|-r+\kappa_{i}}} \\
& \times \prod_{i=k}^{n} \frac{\left(t u_{i} / u_{0} ; q\right)_{\kappa_{i}}}{\left(q u_{i} / u_{0} ; q\right)_{\kappa_{i}}} \prod_{1 \leq i<j \leq n} \frac{\left(q u_{i} / u_{j} ; q\right)_{\kappa_{i}-\kappa_{j}}}{\left(t u_{i} / u_{j} ; q\right)_{\kappa_{i}-\kappa_{j}}} \frac{\left(u_{i} / u_{j} ; q\right)_{\kappa_{i}-\kappa_{j}}}{\left(q u_{i} / t u_{j} ; q\right)_{\kappa_{i}-\kappa_{j}}}
\end{aligned}
$$


together with the pair of mutually inverse matrices $\left(f_{\beta \kappa}\right)$ and $\left(g_{\kappa \gamma}\right)$ as defined in [3, Theorem 2.7].

Several elementary manipulations of $q$-shifted factorials eventually lead to the result in the desired form. To give a sample (concentrating only on the products over $\prod_{1 \leq i<j \leq n}$ of $q$-shifted factorials), we use the simplification

$$
\begin{aligned}
& \frac{\left(q^{\kappa_{i}-\kappa_{j}+1} u_{i} / t u_{j} ; q\right) \beta_{i}-\kappa_{i}}{\left(q^{\kappa_{i}-\kappa_{j}+1} u_{i} / u_{j} ; q\right) \beta_{i}-\kappa_{i}} \frac{\left(q^{\kappa_{i}-\beta_{j}} t u_{i} / u_{j} ; q\right)_{\beta_{i}-\kappa_{i}}}{\left(q^{\kappa_{i}-\beta_{j}} u_{i} / u_{j} ; q\right) \beta_{i}-\kappa_{i}} \\
& \quad \times \frac{\left(q u_{i} / u_{j} ; q\right) \beta_{i}-\beta_{j}}{\left(t u_{i} / u_{j} ; q\right) \beta_{i}-\beta_{j}} \frac{\left(u_{i} / u_{j} ; q\right)_{\beta_{i}-\beta_{j}}}{\left(q u_{i} / t u_{j} ; q\right)_{\beta_{i}-\beta_{j}}} \frac{\left(t u_{i} / u_{j} ; q\right)_{\kappa_{i}-\kappa_{j}}}{\left(q u_{i} / u_{j} ; q\right)_{\kappa_{i}-\kappa_{j}}} \frac{\left(q u_{i} / t u_{j} ; q\right)_{\kappa_{i}-\kappa_{j}}}{\left(u_{i} / u_{j} ; q\right)_{\kappa_{i}-\kappa_{j}}} \\
& =\frac{\left(q u_{i} / t u_{j} ; q\right)_{\beta_{i}-\kappa_{j}}}{\left(q u_{i} / u_{j} ; q\right) \beta_{i}-\kappa_{j}} \frac{\left(t u_{i} / u_{j} ; q\right)_{\kappa_{i}-\beta_{j}}}{\left(q u_{j} ; q\right)_{\kappa_{i}-\beta_{j}}} \frac{\left(q u_{j} ; q\right)_{\beta_{i}-\beta_{j}}}{\left.\left(t u_{i} / u_{j} ; q\right)_{\kappa_{i}-\kappa_{j}} ; q\right)_{\beta_{i}-\beta_{j}}} \frac{\left(u_{i} / u_{j} ; q\right)_{\kappa_{i}-\kappa_{j}}}{\left(u^{\beta_{i}-\beta_{j}+1} u_{i} / t u_{j} ; q\right)_{\beta_{j}-\kappa_{j}}} \frac{\left(q^{\kappa_{i}-\beta_{j}} t u_{i} / u_{j} ; q\right)_{\beta_{j}-\kappa_{j}}}{\left(q^{\kappa_{i}-\beta_{j}} u_{i} / u_{j} ; q\right)_{\beta_{j}-\kappa_{j}}}
\end{aligned}
$$

in the computation of $f_{\beta \kappa}$ in the lemma.

\section{References}

1. Jing, N.H., Józefiak, T.: A formula for two-row Macdonald functions. Duke Math. J. 67, 377-385 (1992)

2. Lassalle, M.: Explicitation des polynômes de Jack et de Macdonald en longueur trois. C. R. Acad. Sci. Paris Sér. I Math. 333, 505-508 (2001)

3. Lassalle, M., Schlosser, M.J.: Inversion of the Pieri formula for Macdonald polynomials. Adv. Math. 202, 289-325 (2006)

4. Macdonald, I.G.: A new class of symmetric functions. Sém. Lothar. Comb. 20, Article B20a (1988)

5. Macdonald, I.G.: Symmetric Functions and Hall Polynomials, 2nd edn. Clarendon, Oxford (1995)

6. Macdonald, I.G.: Symmetric Functions and Orthogonal Polynomials. University Lecture Series, vol. 12. Am. Math. Soc., Providence (1998)

7. Macdonald, I.G.: Orthogonal polynomials associated with root systems. Sém. Lothar. Comb. 45, Article B45a (2000)

8. Macdonald, I.G.: Affine Hecke Algebras and Orthogonal Polynomials. Oxford University Press, Oxford (2003)

9. Perelomov, A.M., Ragoucy, E., Zaugg, P.: Quantum integrable systems and Clebsch-Gordan series: II. J. Phys. A Math. Gen. 32, 8563-8576 (1999) 